\title{
In vitro pyrogen test-A new test method for solid medical devices
}

\author{
Francesca Mazzotti, ${ }^{1}$ Julia Beuttler, ${ }_{1}^{1}$ Richard Zeller, ${ }^{2}$ Ulrich Fink, ${ }^{2}$ Stefanie Schindler, ${ }^{1}$ \\ Albrecht Wendel, ${ }^{1}$ Thomas Hartung, ${ }^{1,3}$ Sonja von Aulock ${ }^{1}$ \\ ${ }^{1}$ Biochemical Pharmacology, University of Konstanz, Germany \\ ${ }^{2}$ B. Braun Melsungen AG, Aesculap, Tuttlingen, Germany \\ ${ }^{3}$ European Centre for the Validation of Alternative Methods, Joint Research Centre, Ispra, Italy
}

Received 6 December 2005; revised 11 April 2006; accepted 15 May 2006

Published online 6 September 2006 in Wiley InterScience (www.interscience.wiley.com). DOI: 10.1002/jbm.a.30922

\begin{abstract}
Medical devices manufactured for implantation into humans must be free of any contamination with viable bacteria. However, remnants of dead bacteria and bacterial components alone may induce an inflammatory immune response. Pyrogen tests for such inflammatory contaminations are generally performed either by determining the content of lipopolysaccharide in rinsing solutions of batch samples by limulus amoebocyte lysate assay, by injecting the rinsing solutions into rabbits or by implanting batch samples into rabbits and measuring change of body temperature. In this study, we show that the in vitro pyrogen test (IPT), which measures the release of the inflammatory cytokine IL-1 $\beta$ in fresh or cryopreserved human whole blood, can be used to assess the pyrogenic contamination of implantable medical devices. This test was used to check neurosurgical implants, namely aneurysm clips, as a proof of principle. Owing to the direct
\end{abstract}

contact of the test material with the blood cells, this test does not require rinsing procedures, which have variable efficacy. The use of human blood ensures the detection of all substances that are pyrogenic for humans and reflects their relative potency. The safety of the products as delivered could be confirmed. The effects of sterilization and depyrogenization procedures on intentional pyrogenic contaminations of samples could be followed. This new application of the already internationally validated method promises to replace further rabbit pyrogen tests. It generates extremely sensitive results with an extended range of detectable pyrogenic contaminants.

Key words: pyrogen testing; human; medical device; whole blood; lipopolysaccharide

\section{INTRODUCTION}

All compounds that induce an inflammatory reaction in the human body are termed "pyrogenic," that is, fever-inducing. ${ }^{1}$ Various structural components of bacteria, yeasts, and molds have been isolated and characterized as pyrogenic. ${ }^{2}$ Organic and inorganic dusts, nanoparticles, and diesel exhaust particles may also induce an inflammatory reaction, although the nature of their pyrogenic components is not yet known. ${ }^{3-5}$

Correspondence to: S. von Aulock; e-mail: Sonja.v-Aulock@ uni-konstanz.de

Contract grant sponsor: German Research Foundation; contract grant number: HA 2567/6-3

Contract grant sponsor: The Fifth Framework Programme; contract grant number: SPIDERMAN G5RD-CT2002-00738

Contract grant sponsors: The Doerenkamp-Zbinden Foundation for Animal-free Research and B. Braun Melsungen AG, Aesculap, Tuttlingen, Germany
When the natural barrier function of the skin and mucous membranes of the human body is compromised or circumvented, for example, during an operation, the tissue comes into direct contact with medical devices (e.g., surgical tools, hypodermic needles, and in some cases, long-term implants, e.g., stents, aneurysm clips, and orthopedic devices). Although these are routinely sterilized by irradiation, autoclaving or gas sterilization, heat-stable pyrogens, which induce an inflammatory reaction resulting in tissue damage to the host or even rejection of the implant, may remain on their surfaces. ${ }^{6,7}$

The registration process of medical devices includes the query of the so-called basic requirements. One of these requirements is pyrogen-free quality. This is proven in the development phase. In most cases, controls accompanying production are not yet required.

Pyrogen tests currently used for medical devices have various drawbacks.

The rabbit pyrogen test requires that sample test materials are implanted into rabbits. A change in body temperature indicates a pyrogenic reaction. One prob- 
lem is that the rabbit may respond differently to some pyrogenic contaminations than a human. ${ }^{8,9}$ Also, this method can only be performed with small implants. The destruction of tissue during implantation into the animal may itself cause an inflammatory reaction that does not necessarily reflect the pyrogenic contamination of the material.

The rabbit pyrogen test may also be performed by injecting a solution gained by rinsing the implant. Generally this procedure is performed using purified water or oil. The extraction efficacy probably depends on temperature, shaking intensity, and storage conditions. However, it is not clear how well pyrogens are solubilized by this procedure. It is also unclear whether adherent pyrogens and pyrogens in solution have different inflammatory properties. We have found strong indications for this regarding the inflammatory capacity of the Gram-positive pyrogen lipoteichoic acid (LTA). Adherence of LTA to a polystyrene surface amplifies its capacity to induce proinflammatory cytokines like TNF $\alpha$ by about three log orders. ${ }^{10}$ Thus, determination of the activity of LTA in a rinsing solution would grossly underestimate its biological potency when adherent to the respective medical device.

A partial alternative method to the latter form of the rabbit pyrogen test is the limulus amoebocyte lysate (LAL) assay. The hemolymph of this horseshoe crab coagulates when brought into contact with lipopolysaccharide (LPS). ${ }^{11}$ The assay can only be performed with liquid samples, thus again only a rinsing solution of the biomaterial can be assessed. ${ }^{1}$ The rinsing solution is prepared with purified water, with or without a detergent (Tween 20). By detecting LPS, the LAL detects the most common and most potent pyrogen known with high sensitivity. However, it does not detect other pyrogens originating from Gram-negative or Gram-positive bacteria, such as lipoproteins, peptidoglycan and lipoteichoic acids, or other pyrogens of a yet unknown chemical nature, and it does not reflect the inflammatory potency of LPS from different bacterial species in a human.,

Pyrogens are recognized in the body by monocytes in the blood and by resident macrophages in the tissues. These cells control the immune response by releasing cytokines. One of the first cytokines released is interleukin (IL)- $1 \beta .{ }^{13}$ IL-1 $\beta$ was initially termed an "endogenous pyrogen," as injection of the recombinant material into a human or animal directly initiates a fever response. IL-1 $\beta$ binds to receptors in the brain where it induces the release of prostaglandin $\mathrm{E}_{2} \quad\left(\mathrm{PGE}_{2}\right)$, which modulates the body's set temperature. ${ }^{14}$

The in vitro pyrogen test (IPT) exploits this reaction to detect the presence of pyrogens in a given sample. ${ }^{15}$ Fresh human whole blood or cryopreserved blood ${ }^{16}$ is brought into contact with the sam- ple and the release of IL-1 $\beta$ is measured by ELISA. A comparison can be made between the IL-1 $\beta$ response to the sample and the response to different concentrations of a standard LPS preparation.

The IPT was originally developed as a safety test for injectable medications. It has recently been validated as an alternative method to the rabbit pyrogen test in an international study and is currently discussed for inclusion into the European Pharmacopoeia. ${ }^{17}$

In the present study, we used the IPT to demonstrate its high sensitivity to detect pyrogenic contaminations on small implantable materials by direct incubation of the sample material with human blood. We were able to demonstrate spike recovery as well as to follow the effects of sterilization and depyrogenization procedures.

\section{MATERIALS AND METHODS}

\section{Sample materials}

LPS from Escherichia coli strain O-113 (EC-6, a generous gift from Dr. S. Poole, National Institute for Biological Standards and Controls, Herts, UK) or Zymosan A (Sigma) were used as pyrogenic stimuli.

As test implants we used industrially manufactured Yasargil aneurysm clips made of titanium alloy (Ti6Al4V according to ISO 5832-3) produced by B. Braun (Aesculap, Tuttlingen, Germany), which are used for intracranial aneurysms (Fig. 1). The clips were supplied in $\gamma$-irradiated condition in two series, cleaned by different standard washing processes established for the cleaning of medical metallic implants. According to limulus amoebocyte lysate

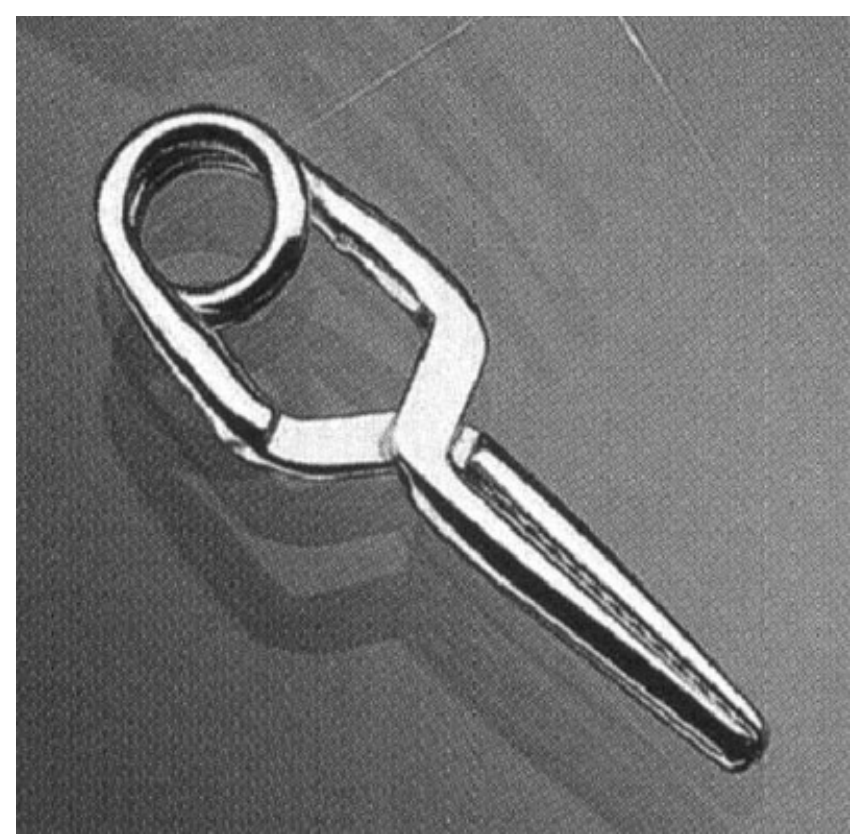

Figure 1. Yasargil titanium aneurysm clip. 
assay measurements done in previous production control steps, the Yasargil clips show values below 1 EEU (endotoxin equivalent unit), well below the accepted safety limit of 20 EEU.

\section{Handling of aneurysm clips}

To obtain a reference point and to evaluate the sensitivity of the in vitro pyrogen test with regard to these medical devices, the aneurysm clips and all forceps used were heattreated for at least $4 \mathrm{~h}$ at $250^{\circ} \mathrm{C}$, a well-accepted and highly effective depyrogenization procedure. Afterwards, some of the clips were contaminated by incubation in a solution containing of $1 \mathrm{ng} / \mathrm{mL}$ LPS overnight. Depyrogenization by sodium hydroxide treatment involved incubation of the clips in $50 \mathrm{~mL}$ of $1 \mathrm{M} \mathrm{NaOH}$ thrice for $20 \mathrm{~min}$ each. Each step was performed in a fresh $50-\mathrm{mL}$ falcon. The clips were then rinsed twice with saline.

\section{Standard washing procedures}

To evaluate the best practice cleaning procedure for the clips, clips taken directly from the production line, which had undergone the standard in-house cleaning process, were compared with clip samples that had undergone an alternative cleaning process.

The standard cleaning process entails that the clips are washed in an ultrasonic bath with an alkaline cleaner ( $\mathrm{pH} 10)$, rinsed with de-ionized water, and dried with hot air. Subsequently, they are washed in alcohol in an ultrasonic bath for $10 \mathrm{~min}$ and packaged in a cleanroom.

The alternative cleaning process was adapted from the well-established cleaning procedure for decontamination of standard surgical instruments in hospitals. In this procedure, the clips are processed for about $90 \mathrm{~min}$ at $55^{\circ} \mathrm{C}$ in the washing step and up to $95^{\circ} \mathrm{C}$ in the disinfection step. In the washing step, the $\mathrm{pH}$-value is in the range of $10-11$.

\section{Whole blood incubation with fresh blood}

Differential white blood cell counts were routinely performed with a Pentra 60 (ABX Diagnostics, Montpellier, France) to exclude acute infections of blood donors.

The sample materials (aneurysm clips) were inserted into 1.5-mL polypropylene reaction vials (Eppendorf) with heat-sterilized forceps together with $1000 \mu \mathrm{L}$ isotonic saline solution (Berlin-Chemie AG) plus $100 \mu \mathrm{L}$ of LPS diluted in saline or $100 \mu \mathrm{L}$ saline as control. A concentration-response curve for LPS $(1000 \mu \mathrm{L}$ isotonic saline plus $100 \mu \mathrm{L}$ of LPS diluted in saline to $12.5,25,50,100,200,400 \mathrm{pg} / \mathrm{mL}$ LPS) was set up in the same type of reaction vials in parallel. This resulted in incubations with a total of 1.25-40 pg LPS per incubation. $100 \mu \mathrm{L}$ of heparinized blood freshly taken from healthy volunteers was added to each reaction vial to reach a final incubation volume of $1200 \mu \mathrm{L}$ (the volume of the aneurysm clips was neglected). The reaction vials were closed and incubated overnight at $37^{\circ} \mathrm{C}$ without agitation (according to the protocol of the Endosafe-IPT kit). Cellfree supernatants were obtained by centrifugation and used for cytokine determination immediately or stored at $-80^{\circ} \mathrm{C}$. IL-1 $\beta$ determination was performed with the Endosafe-IPT kit.

\section{Whole blood incubation with cryopreserved blood}

Cryopreserved blood was prepared as described. ${ }^{16}$ Briefly, the heparinized blood was precooled in ice water for $15 \mathrm{~min}$. Clinical grade dimethylsulfoxide (DMSO, Waco Chemicals) was added to the blood in small amounts to a final concentration of $10 \%$ under constant gentle agitation. Blood was pipetted as $4 \mathrm{~mL}$ aliquots into precooled cryotubes (Nunc) and cooled down to $-5^{\circ} \mathrm{C}$ at a rate of $1^{\circ} \mathrm{C}$ / min in a Nicool Plus PC (Air Liquide). The temperature in the freezing chamber was set to $-30^{\circ} \mathrm{C}$. The crystallization temperature was $-12^{\circ} \mathrm{C}$. When this temperature was reached, the blood was cooled down to $-40^{\circ} \mathrm{C}$ at a rate of $2^{\circ} \mathrm{C} / \mathrm{min}$. The blood was given $120 \mathrm{~s}$ to stabilize before being cooled down to $-120^{\circ} \mathrm{C}$ at a rate of $10^{\circ} \mathrm{C} / \mathrm{min}$. After freezing, the tubes were removed from the freezer and stored in the vapor phase of liquid nitrogen (Air Liquide). After thawing for $30 \mathrm{~min}$ at $37^{\circ} \mathrm{C}$, the blood was used essentially as described earlier for fresh blood. Instead of incubation in saline solution, the cryopreserved blood was incubated in RPMI 1640 (BioWhittaker, Verviers, Belgium).

\section{RESULTS}

\section{Evaluation of pyrogenic activity of heat-depyrogenized clips}

To evaluate the basal pyrogenic activity of the aneurysm clip material and to evaluate the sensitivity of the IPT in this setting, aneurysm clips were completely depyrogenized by heat-treatment, then employed in the IPT, and spiked with $100 \mu \mathrm{L}$ saline containing no $(0 \mathrm{pg}), 5 \mathrm{pg}$ or $40 \mathrm{pg}$ LPS from E. coli $\mathrm{O} 113$ for $2 \mathrm{~h}$ before addition of fresh blood and incubation overnight (Fig. 2). No IL-1 $\beta$ was released in response to the uncontaminated clips. The IL-1 $\beta$ release in response to the clips spiked with $5 \mathrm{pg}$ and 40 pg LPS was comparable (i.e., between $50 \%$ and $200 \%$ spike retrieval) to IL-1 $\beta$ release in response to the same amount of LPS in the absence of the clips (marked by horizontal lines in the figure). Thus, the clip material (titanium) does not interfere with the test.

\section{Recovery of LPS, Zymosan A and manual contamination on heat-sterilized clips}

To demonstrate that the IPT can detect different types of contamination on the titanium alloy, clips were spiked with the yeast extract Zymosan A or were manually contaminated by handling by numerous lab workers not wearing gloves. 


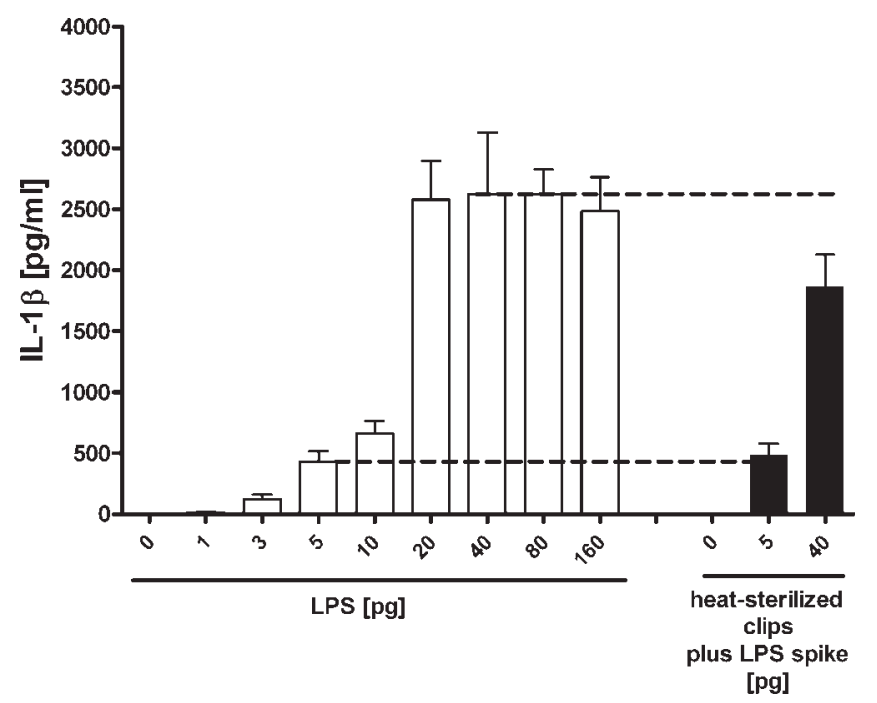

Figure 2. Heat-sterilized titanium clips can be tested by IPT. Clips were sterilized at $250^{\circ} \mathrm{C}$ for $4 \mathrm{~h}$, and then incubated with 0,5 , or 40 pg LPS in saline. In parallel, a concentration response curve of LPS was set up and $100 \mu \mathrm{L}$ fresh human whole blood was added to result in a final reaction volume of $1200 \mu \mathrm{L}$. IL-1 $\beta$ was measured in the supernatant after $24 \mathrm{~h}$ incubation at $37^{\circ} \mathrm{C}$. Data are means \pm SEM of blood from four donors. The horizontal line indicates IL-1 $\beta$ release by the respective standard LPS concentration without a clip. According to the standard operating procedure of the IPT, if the IL- $1 \beta$ release in the sample is at least $50 \%$ of the release in the respective standard LPS concentration, interference is excluded.

Only microgram quantities of Zymosan A induced IL-1 $\beta$ release in human whole blood; however, cytokine induction was in the same order of magnitude in the presence and in the absence of the clips, indicating spike retrieval also of this non-LPS pyrogen

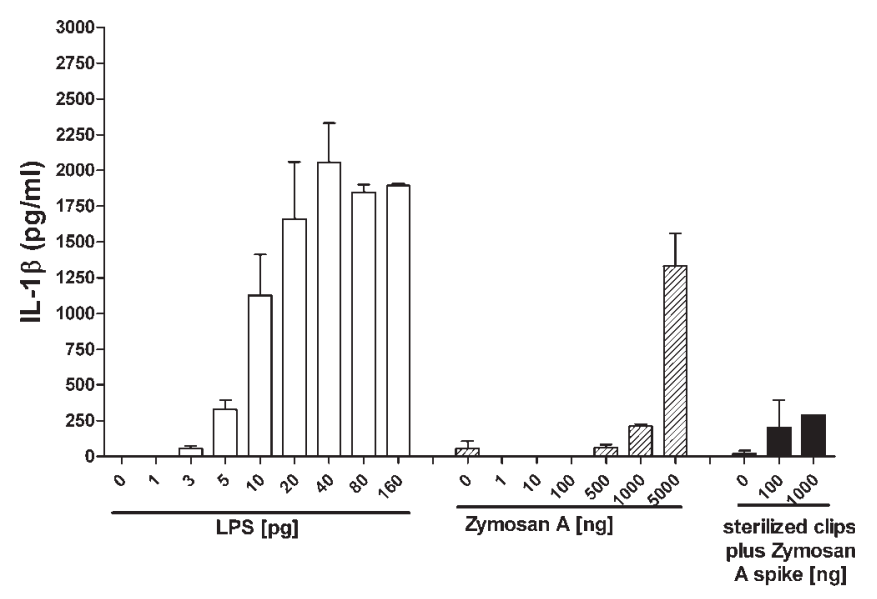

Figure 3. IL-1 $\beta$ induction by Zymosan $A$ in the absence or presence of titanium clips. Whole blood incubation with given amounts of Zymosan $\mathrm{A}$ in a total reaction volume of $1200 \mu \mathrm{L}$ in the presence or absence of titanium clips. Data are means \pm SEM of blood from four donors.

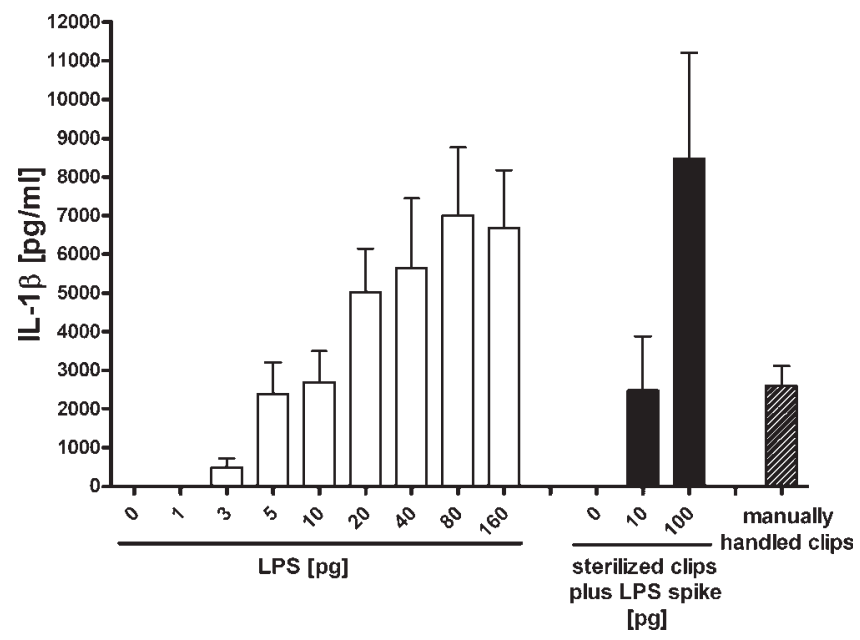

Figure 4. IL-1 $\beta$ induction by manually handled titanium clips. Titanium clips were contaminated by manual handling by laboratory workers without gloves or with 10 and 100 pg LPS and IL-1 $\beta$ release was measured by ELISA. Data are means \pm SEM of blood from three donors.

(Fig. 3). Touching of the clips led to pyrogenic contamination comparable to that of $10 \mathrm{pg}$ LPS, that is, 0.1 EEU, in the absence of the clip (Fig. 4).

\section{Depyrogenization attempts with $\mathrm{NaOH}$ or by rinsing with water}

Next to depyrogenization by dry heat treatment, incubation in sodium hydroxide is known to destroy pyrogens effectively. Clips were contaminated with 100 pg LPS, then incubated in sodium hydroxide and rinsed extensively with pyrogen-free water. Alternately, the clips were contaminated and then only rinsed with water without contact with sodium hydroxide. Figure 5 demonstrates that the contamination was completely destroyed by sodium hydroxide but that rinsing with water alone (one way to prepare an extract for the LAL test) did not readily solubilize the contamination from the clips.

\section{Evaluation of contamination levels during production}

To evaluate the aneurysm clip production process, which does not involve heat-sterilization procedures, clips taken directly from the end of the company's standard production line (including the standard cleaning procedures) were employed in the IPT (Fig. 6) with and without LPS spikes. The clips displayed a low but significant pyrogenic activity equivalent to between 10 and 20 pg LPS, that is, between 0.1 and 0.2 EEU (endotoxin equivalent units) per clip. This activity lies well below the currently accepted limit of 20 EEU per implant. Interestingly, the pyrogenic activity appeared to synergize 


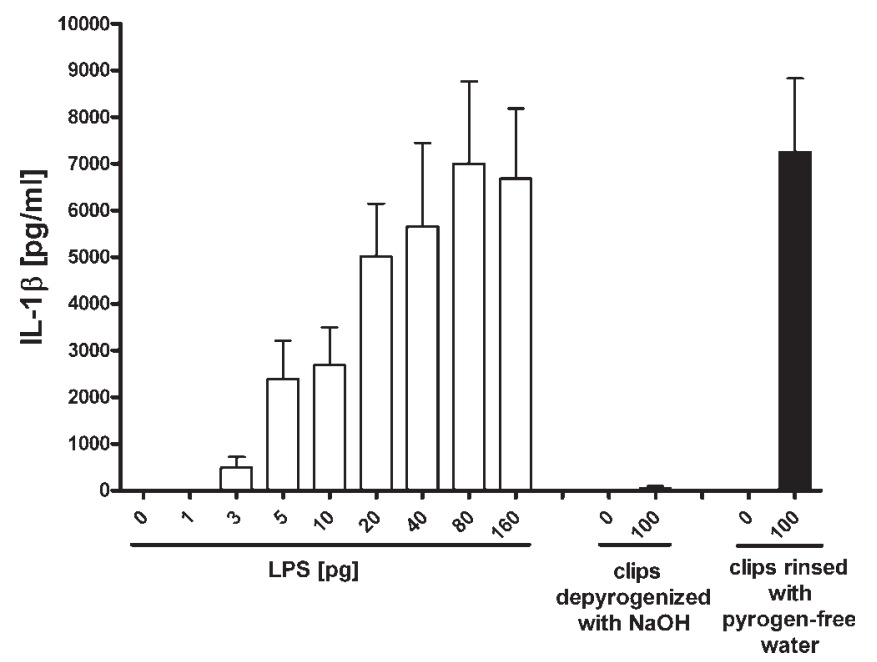

Figure 5. Depyrogenization of LPS-contaminated titanium clips with sodium hydroxide in comparison to rinsing with pyrogen-free water. Clips were contaminated by incubation with $100 \mathrm{pg}$ LPS. Then clips were incubated with $1 \mathrm{M} \mathrm{NaOH}$ three times for $20 \mathrm{~min}$ and rinsed twice with saline, or they were merely rinsed excessively with pyrogen-free water. IL- $1 \beta$ release was determined by ELISA. Data are means \pm SEM of blood from three donors.

with the LPS spike of $5 \mathrm{pg}$, indicating that the residual contamination may not stem from LPS.

\section{Evaluation of a different cleaning procedure for production}

A second batch of aneurysm clips, which had been processed by an alternative cleaning procedure es-

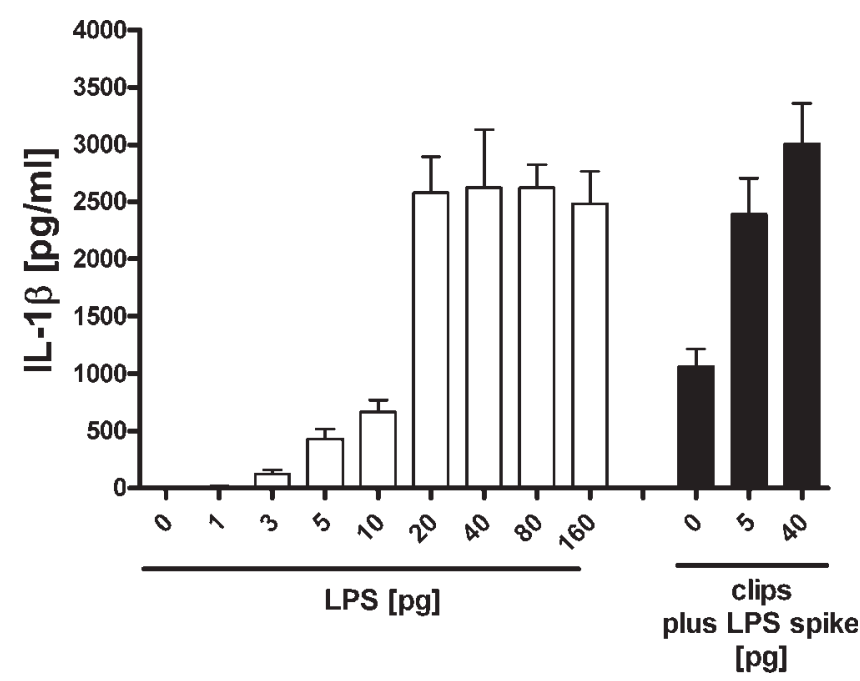

Figure 6. Low contamination of titanium clips during production. Titanium clips packaged for sale were employed directly in the whole blood assay in the presence or absence of LPS. IL-1 $\beta$ release was measured by ELISA. Data are means \pm SEM of blood from four donors.

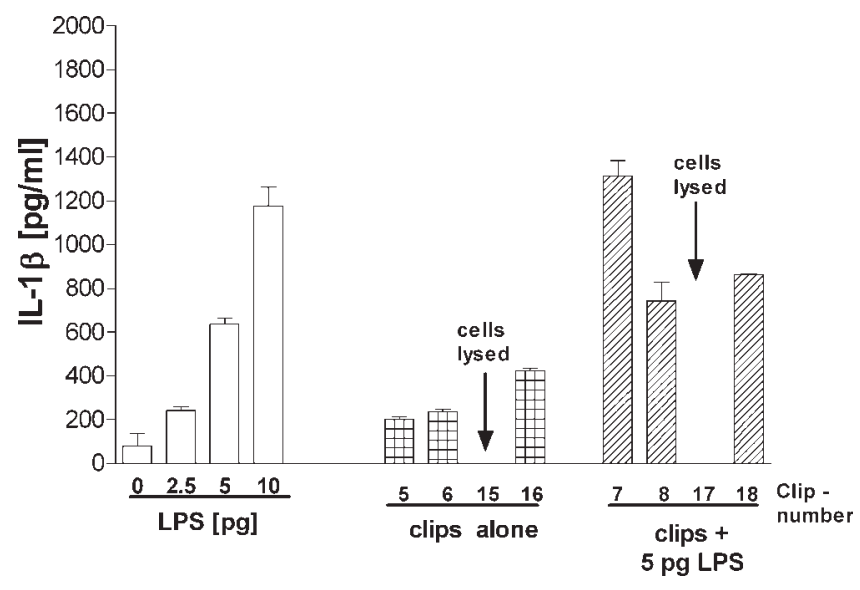

Figure 7. Effect of a new cleaning procedure on the basal contamination of clips during production. Titanium clips that had been washed with an alternative cleaning procedure were incubated in the whole blood assay in the presence or absence of LPS. IL-1 $\beta$ release was measured by ELISA. Data are triplicates of blood from one donor for each clip. Erythrocytes lysed during the incubation procedure where indicated.

tablished for the decontamination of standard surgical instruments in hospitals, was tested in the IPT (Fig. 7). Although the basal contamination of the clips was slightly lower than that measured in Figure 6, residues on some of the clips caused lysis of the erythrocytes in the blood incubation (clips 15 and 17) and interfered with IL-1 $\beta$ measurement.

\section{Comparison of pyrogen detection using fresh with cryopreserved blood}

Clips were spiked with 5 pg LPS and incubated with fresh blood or cryopreserved blood overnight (Fig. 8). Although the cryopreserved blood generally releases a greater quantity of IL-1 $\beta$ than fresh blood, the sensitivity of both assays was the same. The LPS spike added to the clips was detected both in the fresh blood and in the cryopreserved blood (criterion: at least $50 \%$ spike recovery).

\section{DISCUSSION}

Until now the IPT is established and validated for the testing of parenterals, that is, liquids. ${ }^{17}$ In this study, we attempted to measure pyrogenic contamination of model medical devices, that is, aneurysm clips, by IPT. To control for possible effects of our reaction vessels and to allow evaluation of spike recovery, a dilution series of a standard LPS preparation was always set up in parallel in the same reaction vessels. We could show that the titanium of which the clips are made does not interfere with the 

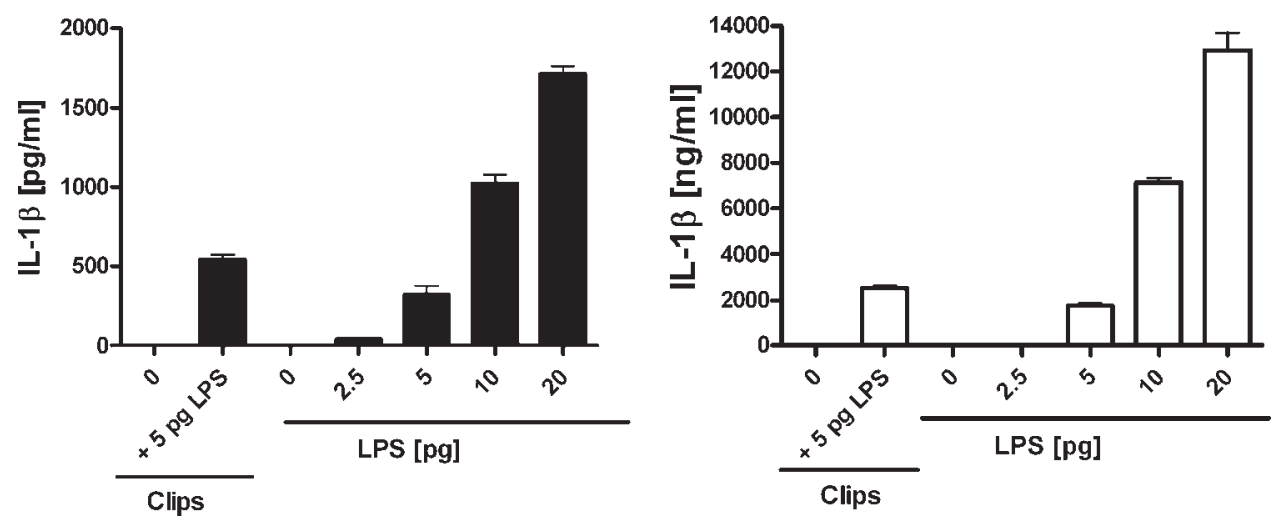

Figure 8. Comparison of the use of fresh and cryopreserved human blood for pyrogen detection on titanium clips. Heatsterilized titanium clips were incubated with or without 5 pg LPS and compared to a concentration response curve to LPS in fresh (A, quintuplicates of one donor) or cryopreserved (B, quintuplicates of a pool of five donors) blood.

detection of pyrogens. As this may not be the case for all conceivable biomaterials, interference will have to be controlled individually for every type of biomaterial tested. Some materials may display inherent pyrogenic activity which would preclude biocompatibility.

The test confirmed the high safety level of the product used as a model medical device. The reason for choosing this product for these investigations was its convenient size: it fits into a $1.5-\mathrm{mL}$ reaction vial. Naturally, other solutions will have to be found for the testing for large or irregularly shaped biomaterials. We have already shown in studies to measure airborne pyrogens that the total reaction volume of diluted blood can easily be increased three-fold ${ }^{18}$ and this can probably be increased even further. Also, the IPT can be performed in closed reaction vials, thus incubation of larger objects in sealed, pyrogen-free plastic bags may be a suitable approach.

Our contamination tests showed that titanium surfaces are able to bind significant amounts of contaminants that are not washed away by mere rinsing with water. This observation has implications for other pyrogen tests. As both the rabbit test and the LAL test are based on rinsing solutions prepared from the sample materials, it appears that these cannot adequately reflect the surface contamination of the material if the pyrogens are not efficiently solubilized, as already suggested by other authors. ${ }^{19}$ In line, it has been shown that the addition of a detergent (Tween 20) during rinsing greatly increases the efficiency of pyrogen extraction. ${ }^{20}$ In further studies, we shall directly compare the pyrogen spike recovery from medical devices attained by these methods.

Depyrogenization of clips with sodium hydroxide treatment or with dry heat was very effective and could be followed with the IPT. As treatment with sodium hydroxide carries the risk of retaining alkaline residues on the surface of the material, the heat- treatment is generally preferred, as long as it does not damage the material.

The experiments performed in this study focused mainly on LPS, the most prominent and a highly potent pyrogen. Previous studies have demonstrated that the IPT can also detect pyrogens other than LPS, including lipoteichoic acids, fungal spores, yeast extracts such as Zymosan A. 17,18,21,22 This was confirmed exemplarily for the application to biomaterials discussed here by using samples contaminated with Zymosan A, manually contaminated samples, and samples taken directly from the production line. Regarding the latter two examples, the nature of the contamination is unknown; however, manual handling represents a realistic source of contamination in a production line. Further studies are required to address how well other known pyrogens are detected on biomaterials and whether their binding to a surface alters their immunostimulatory capacity. The read-out, that is, IL- $1 \beta$ release by blood, does not allow conclusions to be drawn on the type of contamination detected; however, it reflects how the human body would react to the contamination.

As the pyrogenic activity detected on the clips was far below the current limits set for implants, it is evident that the test can be used to evaluate different cleaning processes due to its high sensitivity. When used during the production process, the IPT can sound a warning before a dangerous contamination level is reached. The limit at which adverse biological reactions in humans occur in response to pyrogen-contaminated medical devices needs to be defined more precisely now that this sensitive direct test method is available, and in some cases the currently accepted limits might be reassessed.

We show that biomaterials may be tested with cryopreserved blood as well as with fresh blood. This is important for users who do not have a constant supply of fresh blood available for the assay. The cryopre- 
served blood can be produced in large batches which undergo quality control including screening for HIV and hepatitis virus. The stability of the cryopreserved blood over a period of months also ensures a high comparability between different experiments. ${ }^{16}$

In human whole blood IL-1 $\beta$ is released mainly by monocytes. Therefore, the individual donor's monocyte count influences the absolute amount of cytokine released. These interindividual differences must be controlled by characterizing the individual donor's response or the response of the cryopreserved blood to a dilution series of standard endotoxin in every test procedure.

Flow cytometric studies have shown that approximately $90 \%$ of the monocytes survive the cryopreservation procedure. ${ }^{16}$ Stimulating fresh or cryopreserved blood from 10 donors individually with 5 pg LPS resulted in coefficients of variation in the absolute IL- $1 \beta$ response of $37 \%$ and $75 \%$ respectively. This larger interindividual variation in case of the cryopreserved blood is reduced by pooling blood from at least five donors before freezing. As shown previously, this results in a cytokine response that represents the average of the response of the individual donors. ${ }^{16}$

Although it is difficult to correlate problems with medical devices with pyrogenic contamination retrospectively and the awareness for their adverse influence may not yet be very high, some reports strongly indicate a role of pyrogens in problematic medical devices, especially those made of or coated with materials of biological origin. ${ }^{7,8,23}$

Taken together, using a model implantable material, we have shown that the IPT can be used to evaluate pyrogenic contaminations on medical devices. This assay could lead to a replacement of the rabbit pyrogen assay for this application and could improve current safety testing of biomaterials.

We thank Silvia Asmus for technical assistance.

\section{References}

1. Vanhaecke E, Pijck J, Vuye A. Endotoxin testing. J Clin Pharm Ther 1987;12:223-235.

2. Henderson B, Poole S, Wilson M. Bacterial modulins: A novel class of virulence factors which cause host tissue pathology by inducing cytokine synthesis. Microbiol Rev 1996;60:316341.

3. Siraganian RP, Baer H, Hochstein HD, May JC. Allergenic and biologic activity of commercial preparations of house dust extract. J Allergy Clin Immunol 1979;64(6 Part 1):526-533.

4. Oberdorster G, Oberdorster E, Oberdorster J. Nanotoxicology: An emerging discipline evolving from studies of ultrafine particles. Environ Health Perspect 2005;113:823-839.

5. White HJ, Garg BD. Early pulmonary response of the rat lung to inhalation of high concentration of diesel particles. J Appl Toxicol 1981;1:104-110.
6. Williams KL. Depyrogenation validation, pyroburden, and endotoxin removal from drugs. In: Swarbrick J, editor. Endotoxins: Pyrogens, LAL Testing and Depyrogenaation. New York: Marcel Dekker; 2001. pp 309-343.

7. Williams D. Endotoxins and medical devices: The significance of dead bacteria. Med Device Technol 2003;14:8-11.

8. Nakagawa $Y$, Murai T, Hasegawa C, Hirata M, Tsuchiya T, Yagami T, Haishima Y. Endotoxin contamination in wound dressings made of natural biomaterials. J Biomed Mater Res B Appl Biomater 2003;66:347-355.

9. Spreitzer I, Fischer M, Hartzsch K, Luderitz-Puchel U, Montag T. Comparative study of rabbit pyrogen test and human whole blood assay on human serum albumin. ALTEX 2002;19 (Suppl 1):73-75.

10. Deininger $S$, Traub S, Aichele D, Rupp T, Hartung T, von Aulock S. Presentation of lipoteichoic acid potentiates its inflammatory activity. Forthcoming.

11. Levin J, Bang FB. The role of endotoxin in the extracellular coagulation of limulus blood. Bull Johns Hopkins Hosp 1964; 115:265-274.

12. Williams KL.Nonendotoxin microbial pyrogens: Lesser endotoxins, superantigens and adventitious agents. In: Swarbrick J, editor. Endotoxins: Pyrogens, LAL Testing and Depyrogenaation. New York: Marcel Dekker; 2001. pp 74-98.

13. Bone RC. The pathogenesis of sepsis. Ann Intern Med 1991; 115:457-469.

14. Dinarello CA. Infection, fever, and exogenous and endogenous pyrogens: Some concepts have changed. J Endotoxin Res 2004; 10:201-222.

15. Hartung $\mathrm{T}$, Wendel A. Detection of pyrogens using human whole blood. In Vitro Toxicol 1996;9:353-359.

16. Schindler S, Asmus S, von Aulock S, Wendel A, Hartung T, Fennrich S. Cryopreservation of human whole blood for pyrogenicity testing. J Immunol Methods 2004;294(1/2):89100.

17. Hoffmann S, Peterbauer A, Schindler S, Fennrich S, Poole S, Mistry Y, Montag-Lessing T, Spreitzer I, Loschner B, van Aalderen $M$, Bos $R$, Gommer $M$, Nibbeling $R$, WernerFelmayer G, Loitzl P, Jungi T, Brcic M, Brugger P, Frey E, Bowe G, Casado J, Coecke S, de Lange J, Mogster B, Naess LM, Aaberge IS, Wendel A, Hartung T. International validation of novel pyrogen tests based on human monocytoid cells. J Immunol Methods 2005;298(1/2):161-173.

18. Kindinger I, Daneshian M, Baur H, Gabrio T, Hofmann A, Fennrich S, von Aulock S, Hartung T. A new method to measure air-borne pyrogens based on human whole blood cytokine response. J Immunol Methods 2005;298:143153.

19. Pfeiffer M. Testing medical disposables using the Limulus Amoebocyte Lysate (LAL) test. Med Device Technol 1990;1: $37-41,51$.

20. Douwes J, Versloot P, Hollander A, Heederik D, Doekes G. Influence of various dust sampling and extraction methods on the measurement of airborne endotoxin. Appl Environ Microbiol 1995;61:1763-1769.

21. Fennrich S, Fischer M, Hartung T, Lexa P, Montag-Lessing T, Sonntag HG, Weigandt M, Wendel A. Detection of endotoxins and other pyrogens using human whole blood. Dev Biol Stand 1999;101:131-139.

22. Schindler S, Hoffmann S, Hennes K, Spreitzer I, Halder M, Brügger P, Frey E, Montag-Lessing T, Löschner B, Hartung T. International validation of pyrogen tests based on cryopreserved human primary blood cells. J Immunol Methods. Forthcoming.

23. Yamamoto K, Noishiki Y, Mo M, Kondo J, Matsumoto A. Unusual inflammatory responses around a collagen-impregnated vascular prosthesis. Artif Organs 1993;17:1010-1016. 